\title{
Emission of Thermally Activated Electrons from Rare Gas Clusters Irradiated with Intense VUV Light Pulses from a Free Electron Laser
}

\author{
T. Laarmann, ${ }^{1, *}$ M. Rusek, ${ }^{2}$ H. Wabnitz ${ }^{3}$ J. Schulz, ${ }^{4}$ A. R. B. de Castro, ${ }^{5}$ P. Gürtler, ${ }^{6}$ W. Laasch,${ }^{6}$ and T. Möller ${ }^{7}$ \\ ${ }^{1}$ Max-Born-Institute, Max-Born Strasse 2a, D-12489 Berlin, Germany \\ ${ }^{2}$ Institute of Physics, Polish Academy of Science, Aleja Lotników 32/46, 02-668 Warsaw, Poland \\ ${ }^{3}$ CEA-SPAM, Bâtiment 522, Centre d'Etudes de Saclay, 91191 Gif-sur-Yvette, France \\ ${ }^{4}$ MAX-lab, Box 118, 22100 Lund, Sweden \\ ${ }^{5}$ LNLS, 13084-971 Campinas SP, Brazil and IFGW-UNICAMP, 13083-970 Campinas SP, Brazil \\ ${ }^{6}$ Deutsches Elektronen-Synchrotron DESY, Notkestrasse 85, 22607 Hamburg, Germany \\ ${ }^{7}$ Technische Universität Berlin, Hardenbergstrasse 36, D-10623 Berlin, Germany
}

(Received 14 January 2005; published 5 August 2005)

\begin{abstract}
The ionization dynamics of Ar and Xe clusters irradiated with intense vacuum ultraviolet light from a free-electron laser is investigated using photoelectron spectroscopy. Clusters comprising between 70 and 900 atoms were irradiated with femtosecond pulses at $95 \mathrm{~nm}$ wavelength $(\sim 13 \mathrm{eV}$ photon energy) and a peak intensity of $\sim 4 \times 10^{12} \mathrm{~W} / \mathrm{cm}^{2}$. A broad thermal distribution of emitted electrons from clusters with a maximum kinetic energy up to $30-40 \mathrm{eV}$ is observed. The observation of relatively low-energy photoelectrons is in good agreement with calculations using a time-dependent Thomas-Fermi model and gives experimental evidence of an outer ionization process of the clusters, due to delayed thermoelectronic emission.
\end{abstract}

DOI: $10.1103 /$ PhysRevLett.95.063402

PACS numbers: $36.40 . \mathrm{Gk}, 52.50 . \mathrm{Jm}$

With the advent of intense femtosecond (fs) laser pulses at optical frequencies and an intensity larger than $10^{15} \mathrm{~W} / \mathrm{cm}^{2}$, a variety of fascinating effects were observed in the field of laser matter interaction [1]. One important result of studies on atoms, molecules, and clusters is the possibility of creating photons with energies much larger than that of the initial laser photon. For instance, the observation of coherent radiation in the water window (below the carbon $K$ edge at $4.4 \mathrm{~nm}$ ) through high-order harmonic generation [2] or $\mathrm{keV} \mathrm{x}$-ray emission from atoms with multiple core vacancies received considerable attention [3]. Often cluster beams, especially rare gas clusters, were chosen as target materials to efficiently convert "lowenergy" laser photons into high-energy interaction products. The reason is twofold. Rare gas clusters comprising several hundreds of atoms can easily be prepared in a supersonic expansion and combine a high absorption coefficient due to their solid density with a high penetration depth of laser light through the cluster beam. After irradiation of the clusters, extremely energetic electrons in the $\mathrm{keV}$ range and highly charged ions with $\mathrm{MeV}$ kinetic energies were observed $[4,5]$. The effect of intensity, pulse duration, and cluster size on the dynamics of the interaction has been studied both experimentally and theoretically; see Refs. [6-10], and references therein. On the other hand, only a little is known about the effect of the laser photon energy [11], particularly in the high-energy limit beyond the pioneering work by Rhodes et al. at $248 \mathrm{~nm}$ using intense fs KrF-laser pulses [12].

The vacuum ultraviolet (VUV) free-electron laser (FEL) at DESY has recently opened the door to a new regime of strong field matter interaction in the VUV spectral range
[13]. Gigawatt light pulses of 30-100 fs length with a wavelength below $100 \mathrm{~nm}$ were focused on $\mathrm{Xe}$ and $\mathrm{Ar}$ cluster beams. Coulomb explosion of clusters resulting in multiply charged ions $\left(\mathrm{Xe}^{8+}\right)$ with $\mathrm{keV}$ kinetic energy were observed using time-of-flight mass spectroscopy after irradiation of the clusters at relatively low laser intensity [14]. A photoemission study can give a complementary and more detailed view of nonlinear processes of atoms and clusters in intense laser fields compared to mass spectroscopy. For example, the observation of very energetic electrons with up to $3 \mathrm{keV}$ at optical laser frequencies supported the description of laser cluster interaction in the socalled nanoplasma model [15]. The fundamental question, which of the concepts from the optical regime to describe absorption and ionization of matter can be taken over to the case of intense VUV pulses, goes far beyond "pure" cluster physics. Photoemission gives a fingerprint, particularly of the ionization mechanism in the short wavelength limit, which has not been addressed experimentally so far.

In this Letter, we present a detailed study of cluster ionization induced by intense VUV-FEL light making use of photoelectron spectroscopy and time-dependent calculation in the Thomas-Fermi model. The rare gas clusters $(\mathrm{Xe}, \mathrm{Ar})$ were prepared in a supersonic gas expansion. The average cluster size $\langle N\rangle$ was tuned within the range between 70 and 900 atoms per cluster by varying the stagnation pressure [16]. The full width at half maximum $\Delta N$ of the size distribution is approximately $\langle N\rangle$. FEL radiation $(h \nu \sim 13 \mathrm{eV})$ with a pulse duration of about $100 \mathrm{fs}$ length and typical pulse energies between 0.3 and $5 \mu \mathrm{J}$ was focused on the cluster beam using an elliptical mirror at grazing incidence. The pulse duration was estimated indi- 
rectly with statistical and spectral methods [17]. By measuring the homogeneous focal spot on a fluorescence screen with a microscope and a CCD camera, a diameter of $20 \mu \mathrm{m}$ was derived. As a result, a peak intensity up to $10^{13} \mathrm{~W} / \mathrm{cm}^{2}$ was estimated. The electrons produced during the laser cluster interaction were detected in the direction of the laser polarization with the microchannel plate of a time-of-flight (TOF) spectrometer. The acceptance angle of the detector was limited to $6^{\circ}$ by an entrance aperture in front of the field-free TOF drift tube. Kinetic energies of electrons ranging from a few $\mathrm{eV}$ up to a few $\mathrm{keV}$ can be analyzed. To increase the collection efficiency for lowenergy electrons, an extraction voltage of $-10 \mathrm{~V}$ was applied between the grounded aperture and a grid mounted in a distance of $12 \mathrm{~mm}$ with the laser focus in between. The measured electron distribution curve $I(t)$ was converted into kinetic energy spectra $I(E)$, recalling that $I(E) \propto$ $t^{3} I(t)$, and corrected with respect to the calculated transmission curve of the spectrometer. Figure 1 shows photoelectron spectra from $\mathrm{Ar}_{300}$ clusters (a), Ar atoms (b), $\mathrm{Xe}_{70}$ clusters (c), and Xe atoms (d) irradiated with VUV pulses at $\sim 4 \times 10^{12} \mathrm{~W} / \mathrm{cm}^{2}$. At this intensity rare gas clusters completely disintegrate into multiply charged atomic fragments by Coulomb explosion [14,18]. The spectra were obtained by averaging over several dozen laser shots within a $\pm 34 \%$ laser energy bin, both with and without gas flow in order to subtract the background. Although the statistical error of the averaged data of a few percent is rather low, the subtracted spectra are noisy at low electron energies up to $2.5 \mathrm{eV}$ (long flight times $t$ ). This is due to uncertainties in the background subtraction of spectra with low signal $I(t)$, which are then converted into the energy scale $\left(t^{3}\right)$ and corrected with the strongly varying transmission curve of the spectrometer.

The VUV-laser cluster interaction results mainly in a broad kinetic energy distribution of photoelectrons, which
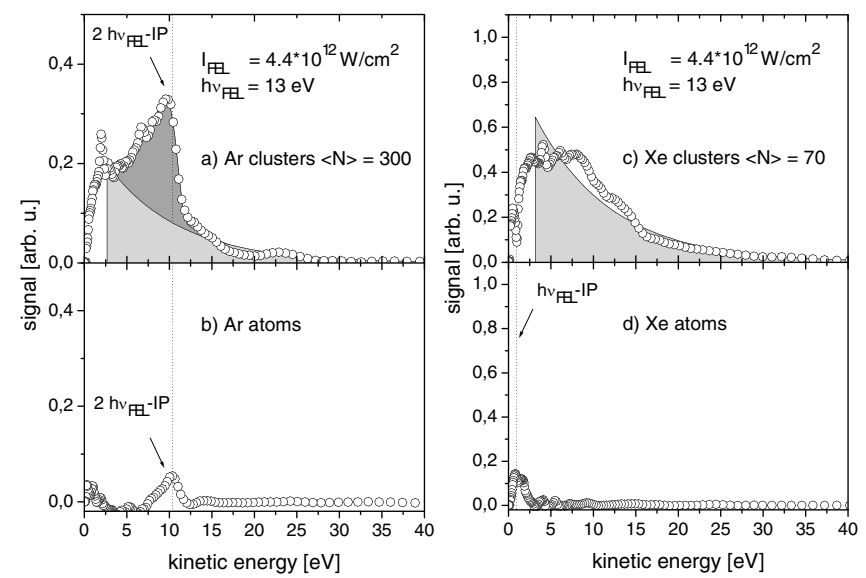

FIG. 1. Photoelectron spectra from $\mathrm{Ar}_{300}$ clusters (a), $\mathrm{Ar}$ atoms (b), $\mathrm{Xe}_{70}$ clusters (c), and $\mathrm{Xe}$ atoms (d) irradiated at $4.4 \times$ $10^{12} \mathrm{~W} / \mathrm{cm}^{2}$. Electrons originating from clusters (light gray shaded) and atoms (dark gray shaded) are indicated. decreases approximately exponentially according to $w\left(E_{\text {kin }}\right)=w_{0} \exp ^{-E_{\text {kin }} / E_{0}}$ with $E_{0} \sim 8.5 \mathrm{eV}(\mathrm{Ar})$ and $E_{0} \sim$ $8.9 \mathrm{eV}(\mathrm{Xe})$ as indicated by the light gray-shaded areas. As pointed out above, the line shape cannot be evaluated properly for $E_{\text {kin }}<2.5 \mathrm{eV}$. In addition to these "thermal" electrons, the Ar cluster spectra exhibit a relatively pronounced asymmetric peak at $\sim 10.4 \mathrm{eV}$ (dark gray). By comparison with the spectrum of a beam of Ar atoms [Fig. 1(b)], this peak can be assigned clearly to two-photon ionization of neutral Ar atoms that are always present in the cluster beam. It should be noticed, that the FEL photon energy is well below the first ionization potential of $\mathrm{Ar}$ atoms $(15.76 \mathrm{eV})$ but above that of $\mathrm{Xe}$ atoms $(12.13 \mathrm{eV})$. Two photons are needed to ionize an Ar atom, whereas one photon is sufficient to ionize Xe. This is indicated by vertical lines in Figs. 1(a)-1(d) [19]. Furthermore, an additional weak structure at $\sim 23 \mathrm{eV}$ appears in the Ar cluster spectrum, which is presumably due to one photon ionization of neutral Ar atoms by the 3rd harmonic of the FEL light. Its contribution is theoretically estimated to be less than $0.1 \%$ of the fundamental power level [20]. An alternative explanation could be above-threshold ionization (ATI), known from infrared laser work. However, the cutoff for ATI at a few $10^{12} \mathrm{~W} / \mathrm{cm}^{2}$ is in the $\mathrm{meV}$ regime due to the small ponderomotive energy at VUV frequencies. The effect of the cluster size on the kinetic energy distribution of emitted photoelectrons is shown in Fig. 2, where the spectrum from $\mathrm{Ar}_{300}$ clusters is compared to that obtained upon irradiation of $\mathrm{Ar}_{900}$ clusters with similar FEL pulses.

A key result is that the maximum kinetic energy of electrons emitted from clusters irradiated with intense VUV pulses is quite low $(<40 \mathrm{eV})$. The energy follows approximately a Boltzmann distribution. This is in striking contrast to $\mathrm{keV}$ electrons emitted from clusters in intense optical laser fields [15]. To shed light on the ionization process, particularly on the thermal distribution of photoelectrons, we performed calculations for Ar clusters using

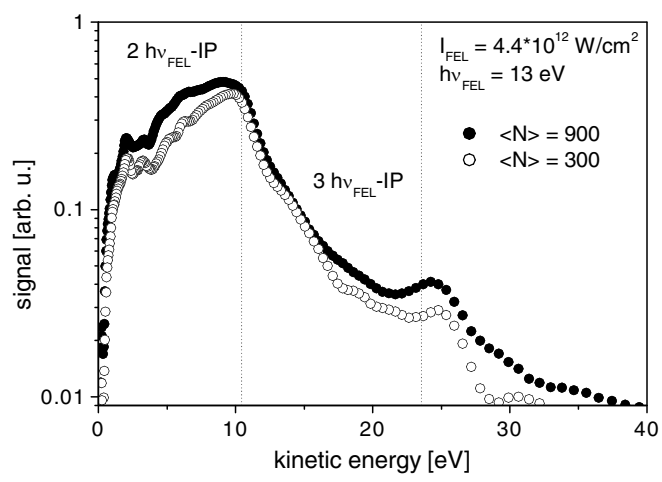

FIG. 2. Photoelectron spectra from Ar clusters comprising 300 (open circles) and 900 atoms (closed circles) plotted in log-linear scale. Contributions from the ionization of atoms, which are always present in the cluster beam, are indicated. 
a time-dependent Thomas-Fermi model [21]. In this model the oscillations of the electron cloud inside the cluster can be viewed as a motion of a fluid characterized by a density and a velocity field. The hydrodynamic equations for the electron density are supplemented by the Newton equations of motion for the positions of the nuclei. The interaction with the laser field is treated within the dipole approximation. The initial, neutral ground state structure is a $\mathrm{Ar}_{55}$ closed-shell icosahedron with an internuclear distance of $3.7 \AA$. The initial electron density distribution was obtained by minimizing the Thomas-Fermi energy functional. For comparison, the calculated kinetic energy spectrum of free electrons emitted in the explosion process of clusters illuminated with VUV pulses is shown in Fig. 3 for a peak intensity of $10^{14} \mathrm{~W} / \mathrm{cm}^{2}$ (a) and $10^{12} \mathrm{~W} / \mathrm{cm}^{2}$ (b). Figure 3 shows clearly the exponential thermal characteristics corresponding to "electron gas temperatures" $E_{0}=6.6 \pm 0.4 \mathrm{eV}$ (a) and $E_{0}=4.2 \pm 0.2 \mathrm{eV}$ (b). With increasing peak intensity the mean kinetic energy of photoelectrons shifts to higher values as intuitively expected. The calculated electron temperature at $10^{12} \mathrm{~W} / \mathrm{cm}^{2}$ differs by a factor of approximately 2 from the experimentally obtained value of $E_{0} \sim 8.5 \mathrm{eV}$. This is presumably a cluster size effect as seen in Fig. 2, since for computational reasons the calculations were performed for $\mathrm{Ar}_{55}$ clusters, whereas $\mathrm{Ar}_{300}$ were studied experimentally.

The photoelectron spectra give direct experimental evidence for thermally activated electron emission in the VUV-laser cluster interaction. Such a process has been suggested recently by simulations, which, however, did not take into account the ion dynamics [22]. The ionization process can be understood in the following way. It is generally accepted that during the absorption of intense fs-laser light by clusters, a highly excited medium is formed. Then the cluster undergoes different phases,

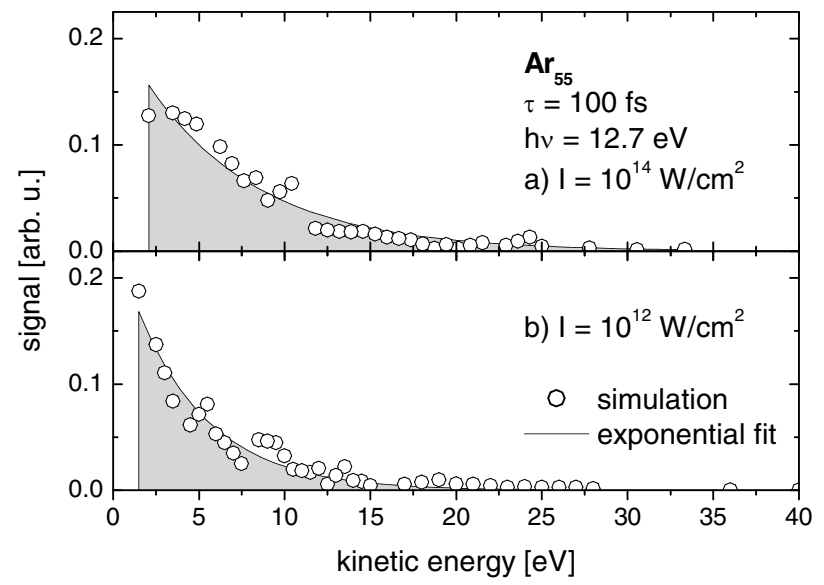

FIG. 3. Simulation of the photoemission spectra from $\mathrm{Ar}_{55}$ clusters irradiated with laser pulses at a peak intensity of (a) $10^{14} \mathrm{~W} / \mathrm{cm}^{2}$ and (b) $10^{12} \mathrm{~W} / \mathrm{cm}^{2}$ using a time-dependent Thomas-Fermi model. Exponential fits of the kinetic energy distributions are indicated (gray shaded). namely, (i) inner ionization of the cluster atoms forming a nanoplasma, (ii) outer ionization of the quasifree electrons from the highly charged cluster, which finally results in (iii) the complete disintegration of the cluster [7]. At optical frequencies, the inner ionization occurs through either multiphoton or field ionization (above-barrier or tunneling). Which process dominates can be estimated with the so-called Keldysh parameter $\gamma$ [23]. If $\gamma$ is smaller than 1 field ionization dominates, whereas for a large Keldysh parameter multiphoton processes control the plasma formation. In case of VUV-laser cluster interaction discussed in this Letter, we are in the multiphoton regime [14]. This is also evident from the two-photon ionization peak of neutral Ar atoms shown in Fig. 1(b). Therefore, at the beginning of the VUV-laser cluster interaction, the inner ionization of the cluster occurs mainly by two-photon (one photon) absorption of the $\mathrm{Ar}(\mathrm{Xe})$ atoms inside the cluster. After a few fs a plasma is formed and most of the produced electrons are bound by strong Coulomb forces to the dense cluster ion core. The energy deposition is then mainly controlled by collisional heating of the plasma [2426], which is more efficient in larger clusters (Fig. 2) and at higher laser intensities (Fig. 3). Quasifree electrons inside the cluster, which gain enough energy to overcome the potential barrier, leave the dense cluster ion core (outer ionization).

Additional information on the ionization dynamics is obtained by calculating the number of emitted (free) electrons as a function of the irradiation time shown in Fig. 4. As input FEL parameters for the simulation, we took those of Fig. 3(b). The plot shows clearly that at the beginning of the VUV pulse, when the plasma has already been formed, almost no electrons are able to leave the cluster due to the strong Coulomb attraction. This behavior is completely different from that at optical frequencies, where a signifi-

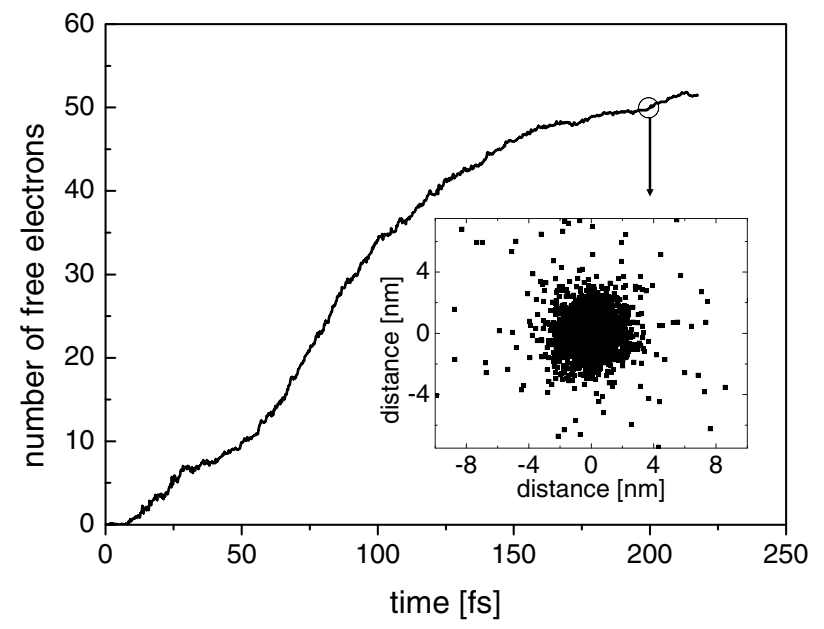

FIG. 4. Calculated number of emitted electrons as a function of the irradiation time. The inset shows a $2 \mathrm{D}$ projection of the pseudoparticles that model the electron density distribution, in a "snapshot" at the end of the pulse. 
cant fraction of electrons from the cluster surface is immediately "pulled out" on the leading edge of the pulse in the direction of the laser polarization by field ionization [15]. Further, it can be seen that a large fraction of electrons is emitted delayed, after the FEL pulse has passed and the expansion of ions has started. For large clusters $(N>$ 1000) we can expect that most electrons are emitted when the pulse is already switched off. The inset of Fig. 4 shows a two dimensional projection of the pseudoparticles modeling the electron density. This "snapshot" after 200 fs clearly shows that electron emission further takes place and that the emission is isotropic. These results can be understood in the following way. According to the calculation, the electrons undergo many scattering events inside the cluster before outer ionization leading to a statistical redistribution of energy among the electronic degrees of freedom [27]. Since the FEL pulse duration is short compared to the characteristic time scale for electron phonon coupling ( $\mathrm{ps}$ ), the transient-electron temperature becomes much larger than the local-lattice temperature by a transfer of laser energy into internal energy of the electron gas. Electrons close to the cluster surface in the highenergy tail of the thermal distribution can escape from the cluster during the Coulomb explosion, i.e., once the Coulomb barrier is significantly lowered due to the cluster expansion. A delayed release of thermally excited electrons follows, which may be called thermoelectronic emission in contrast to thermionic emission, where the electronic and nuclear degrees of freedom are in equilibrium [28].

In conclusion, the interaction of rare gas clusters comprising between 70 and 900 atoms with intense fs VUVlaser pulses was investigated with photoelectron spectroscopy. At a peak intensity of $\sim 4 \times 10^{12} \mathrm{~W} / \mathrm{cm}^{2}$, the kinetic energy distribution of emitted electrons from clusters decreases exponentially up to a kinetic energy of $30-40 \mathrm{eV}$. A characteristic electron temperature below $10 \mathrm{eV}$ is obtained. Calculations using a time-dependent ThomasFermi model, taking into account collisional heating effects, support the description of ionization as thermoelectronic emission. These low-energy electrons are released temporally delayed without any memory of the laser polarization. These findings are in striking contrast to that at optical frequencies, namely, (i) field ionization of surface electrons on the leading edge of the laser pulse and (ii) delayed emission of $\mathrm{keV}$ bulk electrons described by the nanoplasma model. The different ionization mechanism also explains why the electrons emitted in VUV-light cluster interactions have much less kinetic energy than at optical frequencies.

We thank the TTF team at DESY. This work was financially supported by the DFG.
*Electronic address: laarmann@ mbi-berlin.de

[1] D. Batani et al., in Atoms, Solids and Plasmas in Super-Intense Laser Fields (Kluwer Academic/Plenum Publishers, New York, 2001).

[2] P. Salieres, in Atoms, Solids and Plasmas in Super-Intense Laser Fields (Ref. [1]), pp. 83-97.

[3] A. McPherson, B. D. Thompson, A. B. Borisov, K. Boyer, and C. K. Rhodes, Nature (London) 370, 631 (1994).

[4] T. Ditmire, J. Zweiback, V.P. Yanovsky, T.E. Cowan, G. Hays, and K. B. Wharton, Nature (London) 398, 489 (1999).

[5] E. Springate et al., Phys. Rev. A 61, 063201 (2000).

[6] I. Last and J. Jortner, Phys. Rev. A 62, 013201 (2000).

[7] V.P. Krainov and M. B. Smirnov, Phys. Rep. 370, 237 (2002).

[8] V. Kumarappan, M. Krishnamurthy, and D. Mathur, Phys. Rev. A 67, 043204 (2003).

[9] C. Siedschlag and J. M. Rost, Phys. Rev. A 67, 013404 (2003).

[10] S. Zamith et al., Phys. Rev. A 70, 011201(R) (2004).

[11] U. Saalmann and J. M. Rost, Phys. Rev. Lett. 89, 143401 (2002).

[12] W. A. Schroeder et al., J. Phys. B 31, 5031 (1998).

[13] V. Ayvazyan et al., Phys. Rev. Lett. 88, 104802 (2002).

[14] H. Wabnitz et al., Nature (London) 420, 482 (2002).

[15] Y.L. Shao et al., Phys. Rev. Lett. 77, 3343 (1996).

[16] U. Buck and R. Krohne, J. Chem. Phys. 105, 5408 (1996).

[17] V. Ayvazyan et al., Eur. Phys. J. D 20, 149 (2002).

[18] T. Laarmann et al., Phys. Rev. Lett. 92, 143401 (2004).

[19] The small peak shift of $\sim 1 \mathrm{eV}$ between photoelectron spectra of atomic and cluster beams, as well as the asymmetric broadening of the peak towards lower energy is presumably due to a space charge effect in the interaction volume, taking into account the spectrometer resolution at $10 \mathrm{eV}$ of $\sim 2 \mathrm{eV}$ (FWHM). The photoelectrons are slowed down by the large number of positively charged ions resulting from the Coulomb explosion of clusters and reach the detector somewhat later, respectively, outside the detection time window in case of $\mathrm{Xe}$ atoms. Furthermore, Ar dimers and small clusters that are always present as admixtures in the beam can contribute to this broadened peak.

[20] E. Saldin (private communication).

[21] M. Rusek, H. Lagadec, and T. Blenski, Phys. Rev. A 63, 013203 (2001).

[22] J. Schulz et al., Nucl. Instrum. Methods Phys. Res., Sect. A 507, 572 (2003).

[23] L. V. Keldysh, Sov. Phys. JETP 20, 1307 (1965).

[24] R. Santra and C. Green, Phys. Rev. Lett. 91, 233401 (2003).

[25] C. Siedschlag and J. M. Rost, Phys. Rev. Lett. 93, 043402 (2004).

[26] D. Bauer, J. Phys. B 37, 3085 (2004).

[27] J. U. Andersen, E. Bonderup, and K. Hansen, J. Phys. B 35, R1 (2002).

[28] D. M. Riffe et al., J. Opt. Soc. Am. B 10, 1424 (1993). 